\title{
Role of short-term follow-up magnetic resonance imaging in the detection of post-operative residual breast cancer
}

\author{
YILI ZHANG and HONGWEN DU
}

\begin{abstract}
Department of Radiology, The First Affiliated Hospital of Xi'an Jiaotong University, Xi'an, Shaanxi 710061, P.R. China
\end{abstract}
Received November 10, 2015; Accepted May 20, 2016

DOI: $10.3892 / \mathrm{mco} .2016 .923$

\begin{abstract}
The aim of the present study was to evaluate the role of short-term follow-up magnetic resonance imaging (MRI) in the detection of postoperative residual breast cancer. A retrospective analysis was performed on 10 patients who were diagnosed with non-malignant breast lesions by preoperative clinical, ultrasound and mammography examinations and intraoperative frozen-section pathology. These patients were finally confirmed as having malignant breast lesions by paraffin-embedded tissue histology and corresponding received second surgeries. Routine MRI, enhancement MRI and echo-planar imaging-diffusion-weighted imaging were performed on the 10 patients within 1 month after the first surgery. All the cases showed a local distortion of mammary architecture revealed by routine MRI and enhancement MRI images. The enhancement characteristics of the 10 cases were as follows: 3 cases featured stippled enhancement, 2 had small nodular enhancement, 1 showed dendritic enhancement, 1 had a ring-shaped enhancement of the cystic wall and 3 had no abnormal enhancement. The lesions of 7 cases had a type-I enhancement curve (progressive enhancement pattern) and 3 cases had a type-II curve (plateau pattern). The lesions of 4 cases had a decreased apparent diffusion coefficient. In total, 4 cases of tumor residue were diagnosed by MRI and the second pathological examination, while in 1 case the tumor residue was misdiagnosed by MRI but confirmed by the second pathological examination. In conclusion, the present study suggested that short-term follow-up MRI may be of value in the diagnosis of postoperative residual breast tumors and may be helpful for surgeons to develop an accurate surgical plan.
\end{abstract}

Correspondence to: Dr Yili Zhang or Dr Hongwen Du, Department of Radiology, The First Affiliated Hospital of Xi'an Jiaotong University, 277 West Yanta Road, Xi'an, Shaanxi 710061, P.R. China E-mail: yilizhang1975@hotmail.com

E-mail: dwendy@126.com

Key words: magnetic resonance imaging, breast, residual cancer

\section{Introduction}

Technological improvements in the field of medical imaging, including mammography, ultrasonography and magnetic resonance imaging (MRI), have markedly enhanced the accuracy for breast cancer diagnosis in the early stage (1-3). However, false-positive and false-negative diagnosis of breast cancer based on imaging remains $(4,5)$. Therefore, intraoperative frozen section diagnosis has a vital role in guiding appropriate therapeutic decision making for breast carcinoma patients and is widely used in the clinic $(6,7)$. However, due to reasons such as poor quality of frozen sections, incorrect sampling procedures and limited experience of pathologists, the average accuracy of diagnoses based on intraoperative frozen pathology was 92.6-98.0\% (8). Misdiagnosis based on intraoperative frozen pathology may lead to the selection of an inappropriate breast surgical procedure, resulting in incomplete tumor resection or unnecessary removal of the whole breast.

False-negative cases require a second surgery as soon as possible in order to remove the residual tumor and ensure tumor-free surgical margins. Prior to the second surgery, the detailed condition of the postoperative breast must be known in order to develop an appropriate surgical plan. For this, a precise breast imaging reexamination is important. Mammography is not suitable for immediate postoperative imaging as compression of the breast may lead to wound dehiscence. Ultrasound examination is also not suitable for postoperative imaging, as the image quality is usually largely affected by local structural disorders and edema in the surgical area. MRI is now widely accepted as the most accurate imaging modality for assessment of breast tumors, as it is capable of detecting unsuspected multifocal/multicentric or contralateral breast cancers and is suitable for guiding therapeutic decisions (9-11). Therefore, MRI was used as the imaging reexamination method in the present study.

\section{Materials and methods}

Patients. Between January 2011 and December 2013, 10 breast cancer patients diagnosed with non-malignant breast lesions by preoperative clinical and imaging examinations, as well as by intraoperative frozen section pathology, but who were finally confirmed as having malignant breast lesions by paraffin-embedded tissue histology, were included in the present study. All the patients were females with an average 


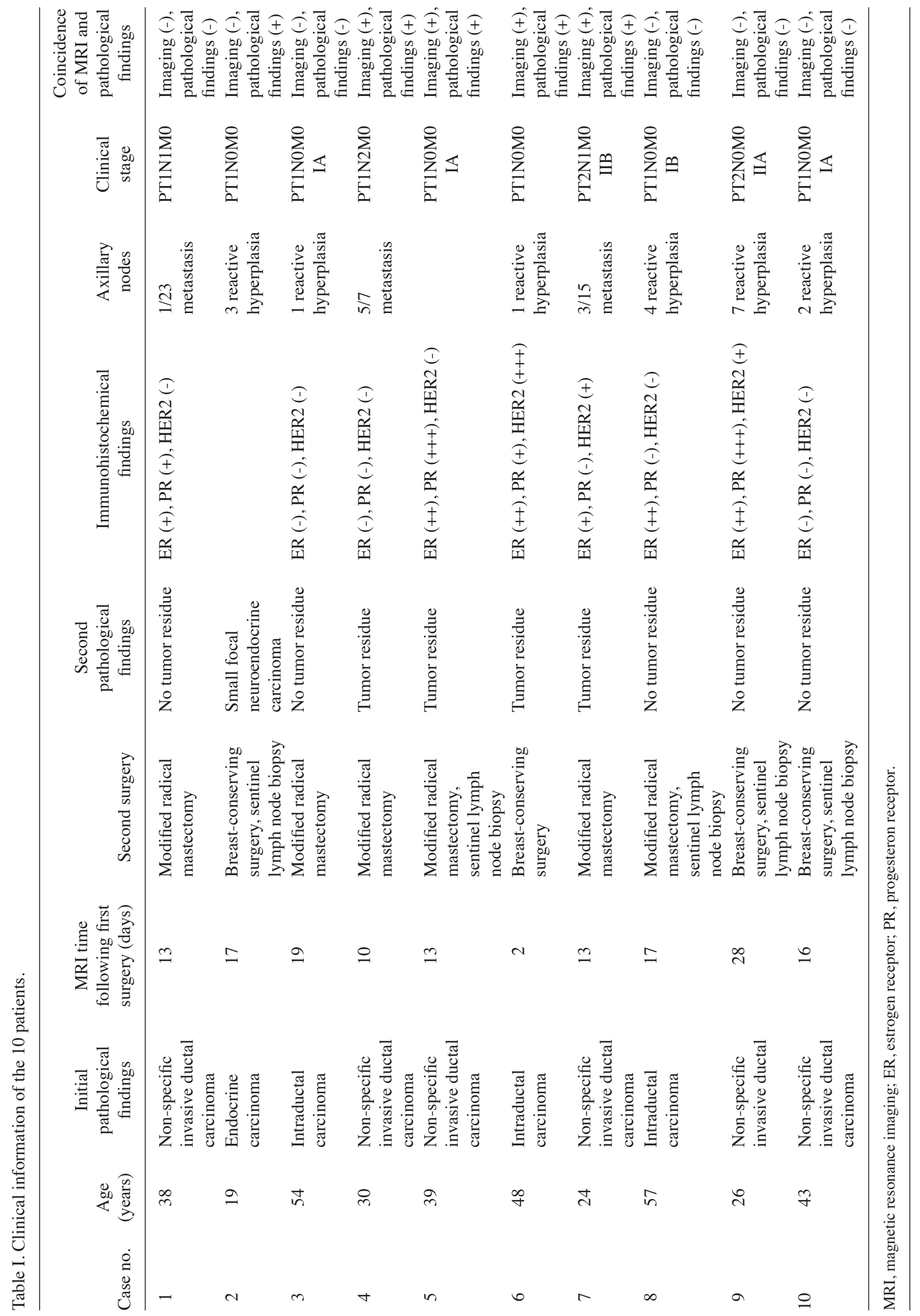


Table II. Breast MRI features of the patients.

\begin{tabular}{llr}
\hline Imaging method & \multicolumn{1}{c}{ Imaging feature } & Cases (n) \\
\hline MRI plain scan & Structural disorder & 10 \\
& Local skin, subcutaneous tissue edema & 10 \\
& Residual cavity & 3 \\
Diffusion-weighted imaging scan & Decrease in apparent diffusion coefficient & 4 \\
MRI dynamic enhancement scan & Stippled enhancement & 3 \\
& Nodular enhancement & 2 \\
& Dendritic enhancement & 1 \\
& Ring-like enhancement of cystic wall & 1 \\
& No abnormal enhancement & 3 \\
& Type I curve & 7 \\
\hline
\end{tabular}

MRI, magnetic resonance imaging.

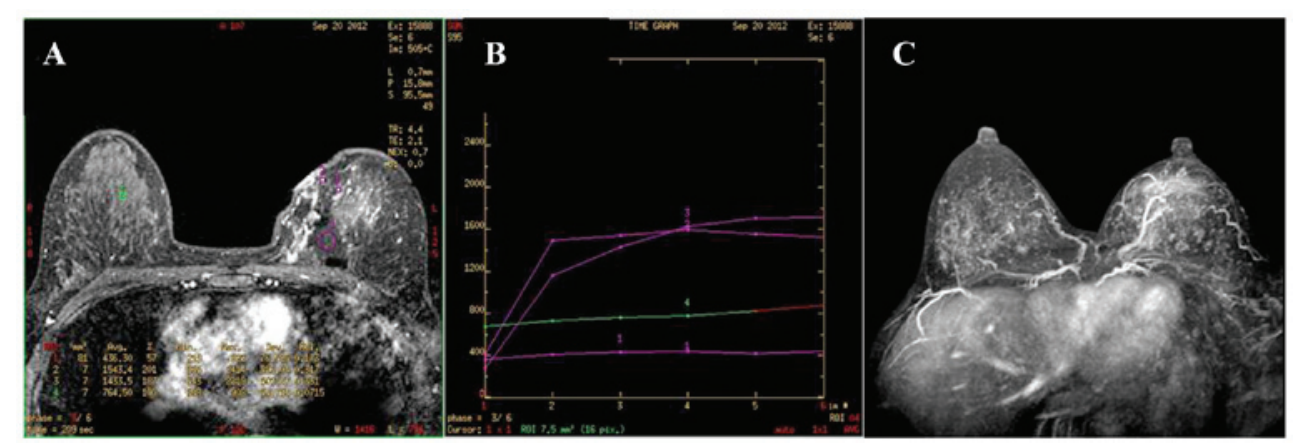

Figure 1. Magnetic resonance images of case no. 6, a 48-year-old with intraductal carcinoma. (A) Vibrant+C T1 sequence with fat suppression showed structural disorder, dendritic enhancement in the medial left breast, as well as thickened skin. (B) Curves of enhancement had a plateau shape (type II), (C) 3D maximum intensity projection reconstruction image showing disordered vasculature which had entered into the lesions in the left breast.

age of $37.8 \pm 12.9$ years (range, $19-57$ years). Six of the cases had non-specific invasive ductal carcinoma, 3 had intraductal carcinoma and 1 had endocrine carcinoma. Clinical data of the patients are listed in Table I. The present study was approved by the Ethics Committee of Xi'an Jiaotong University (Xi'an, China) and all patients gave written informed consent prior to enrolment in the study.

MRI acquisition and analysis. All patients were subjected to MRI examination between days 2 and 28 after the first surgery with an average time interval of 15 days.

MRI was performed using a 3.0-T system (GE Signa HDxt; GE Healthcare, Milwaukee, WI, USA). All patients were examined in a prone position using a double breast coil. An axial fat-suppressed T2-weighted short-TI inversion recovery sequence was performed with the following parameters: Repetition time(TR)/echotime(TE), $8,800.00 \mathrm{msec} / 33.88 \mathrm{msec}$; field of view, $320.00 \mathrm{~mm}$; matrix size, 512x512; and slice thickness, $4 \mathrm{~mm}$ with a 1-mm gap. An axial T1 sequence was performed with the following parameters: TR/TE, $580 \mathrm{msec} / 7.20 \mathrm{msec}$; field of view, $320.00 \mathrm{~mm}$; matrix size, $512 \times 512$; and slice thickness, $4 \mathrm{~mm}$ with a 1-mm gap. An axial echo-planar imaging (EPI)-diffusion-weighted imaging
(DWI) sequence $(b=1,000)$ was performed with the following parameters: TR/TE, $6,000 \mathrm{msec} / 69.60 \mathrm{msec}$; field of view, $320.00 \mathrm{~mm}$; matrix size, 256x256; and slice thickness, $4 \mathrm{~mm}$ with a 1-mm gap. An axial Vibrant $+\mathrm{C}$ sequence was performed with the following parameters: TR/TE, $4.29 \mathrm{msec} / 2.10 \mathrm{msec}$; field of view, $320.00 \mathrm{~mm}$; matrix size, $512 \times 512$; and slice thickness, $1.4 \mathrm{~mm}$ with a $0.7-\mathrm{mm}$ gap. The contrast agent (Omniscan ${ }^{\circledR}$; GE Healthcare, Cork, Ireland; $0.1 \mathrm{mmol} / \mathrm{kg}$ body weight) was manually injected at the beginning of the 5th acquisition, followed by $10 \mathrm{cc}$ saline to flush in all contrast medium. Two experienced radiologists independently reviewed the images using ImageJ 1.44p software (National Institutes of Health, Bethesda, MD, USA).

\section{Results}

MRI analysis. The breast MRI features of the 10 patients are summarized in Table II. All the cases showed a local mammary architecture distortion in the routine MRI and in the enhancement MRI. The enhancement characteristics of the 10 cases were as follows: 3 cases showed stippled enhancement, 2 had small nodular enhancement, 1 featured dendritic enhancement (Fig. 1), 1 showed a ring-shaped 


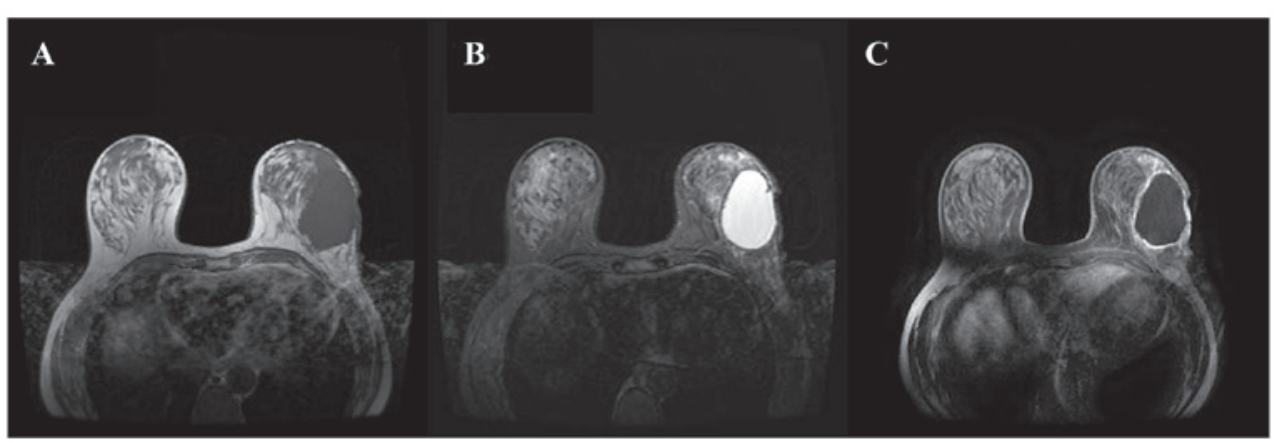

Figure 2. Magnetic resonance images of case no. 8, a 57-year-old with intraductal carcinoma. (A and B) T1-weighted and T2 fat suppression showed a residual cavity in the lateral left breast, a homogeneous T1-type hypointense signal and a homogeneous T2 fat suppression hyperintense signal. The adjacent skin tissue was defective. (C) Vibrant+C T1 sequence with fat suppression showed a ring-like enhancement of the residual cavity wall.

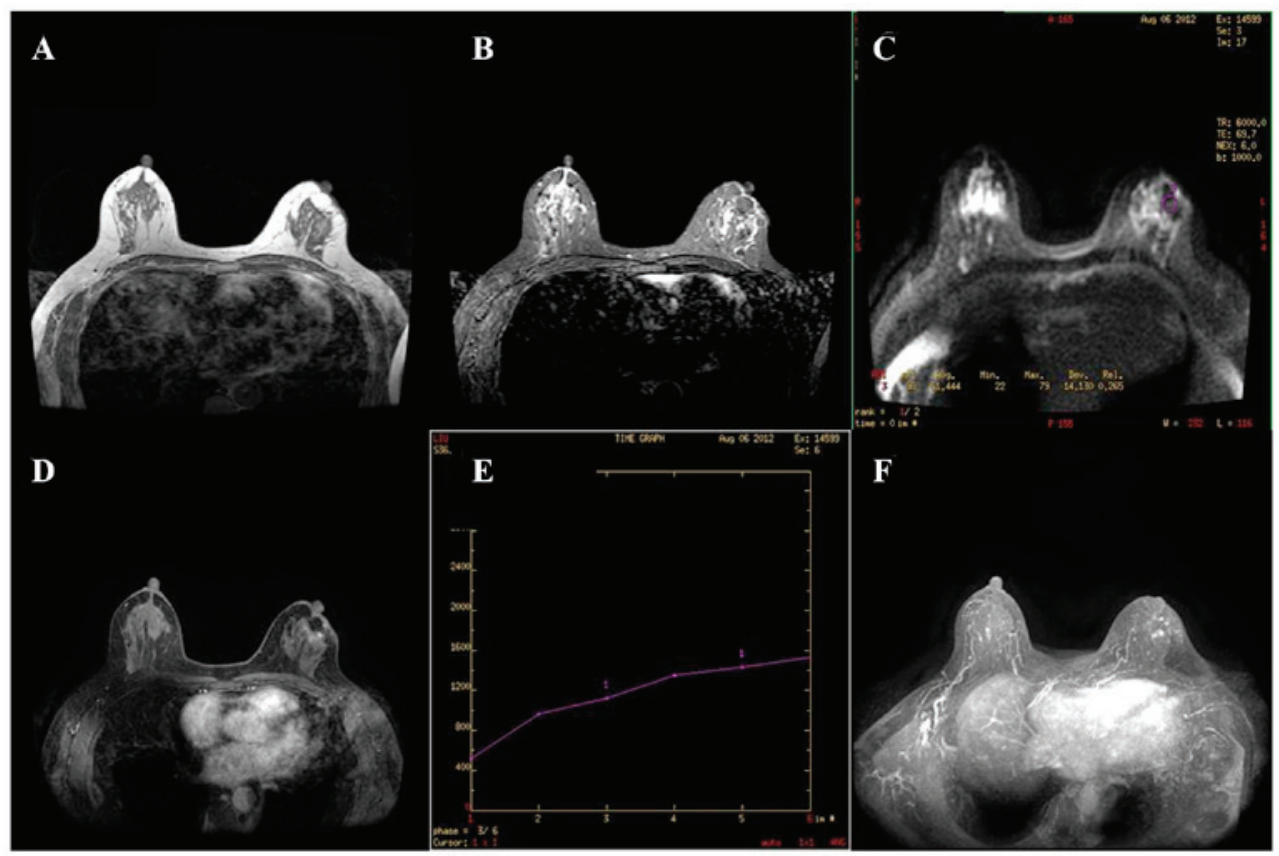

Figure 3. Magnetic resonance images of case no. 3, a 54-year-old with intraductal carcinoma. (A and B) T1-weighted and T2 fat suppression showed that the left breast was smaller than the right breast and that its lateral section had structural disorders. A patchy hypointense T1 signal and a hyperintense T2 fat suppression signal were observed, and the adjacent skin tissue was thickened. (C) Echo-planar imaging-diffusion-weighted imaging (DWI) showed a hyperintense lesion in the DWI $(\mathrm{b}=1,000)$ and the apparent diffusion coefficient was $0.77 \times 10^{-3} \mathrm{~mm}^{2} / \mathrm{sec}$. (D and F) Vibrant $+\mathrm{C}$ T1 with fat suppression and 3D maximum intensity projection reconstruction images showed structural disorder and nodular enhancement. (E) The curve of enhancement had a progressive pattern (type I).

enhancement of the cystic wall (Fig. 2) and 3 had no abnormal enhancement. The lesions of 7 cases had a type-I enhancement curve (progressive enhancement pattern) and 3 had a type-II enhancement curve (plateau pattern). Regarding the apparent diffusion coefficients (ADCs) of the 10 patients, 4 were found to be abnormally decreased according to the threshold value of $1.2 \pm 0.25 \times 10^{-3} \mathrm{~mm}^{2} / \mathrm{sec}$ (12), with resulting ADCs of $0.77 \times 10^{-3} \mathrm{~mm}^{2} / \mathrm{sec}$ (Fig. 3), $0.96 \times 10^{-3} \mathrm{~mm}^{2} / \mathrm{sec}$, $0.88-1.07 \times 10^{-3} \mathrm{~mm}^{2} / \mathrm{sec}$ (Fig. 4) and $1.18 \times 10^{-3} \mathrm{~mm}^{2} / \mathrm{sec}$ respectively.Furthermore, 1 swollenaxillarylymphnodewasfoundinthe armpit of the lesion side with an ADC of $0.7 \times 10^{-3} \mathrm{~mm}^{2} / \mathrm{sec}$ (Fig. 5).

Based on the morphological features and curves of the enhancement MRI, as well as the ADC values, the presence of residual breast cancer was evaluated. The results demonstrated that tumor residues were present in 4 cases and 1 axillary lymph node metastasis was detected by MRI and the second pathological analysis, while in only 1 case, the tumor residue was misdiagnosed by MRI but confirmed by the second pathological analysis.

All of the patients received corresponding second surgeries. Among them, 4 underwent breast-conserving surgery and 6 underwent modified radical mastectomy.

\section{Discussion}

With the development of cytological techniques for determining the nature of sono- or mammographically diagnosed breast abnormalities, percutaneous image-guided core needle biopsy has become an alternative to surgical biopsy for the histological assessment of breast lesions (13). However, intraoperative frozen section pathology is widely used in numerous countries, including China. In general, frozen section pathology examination is a requisite during surgery for all patients with diseases of the breast and surgeons select 


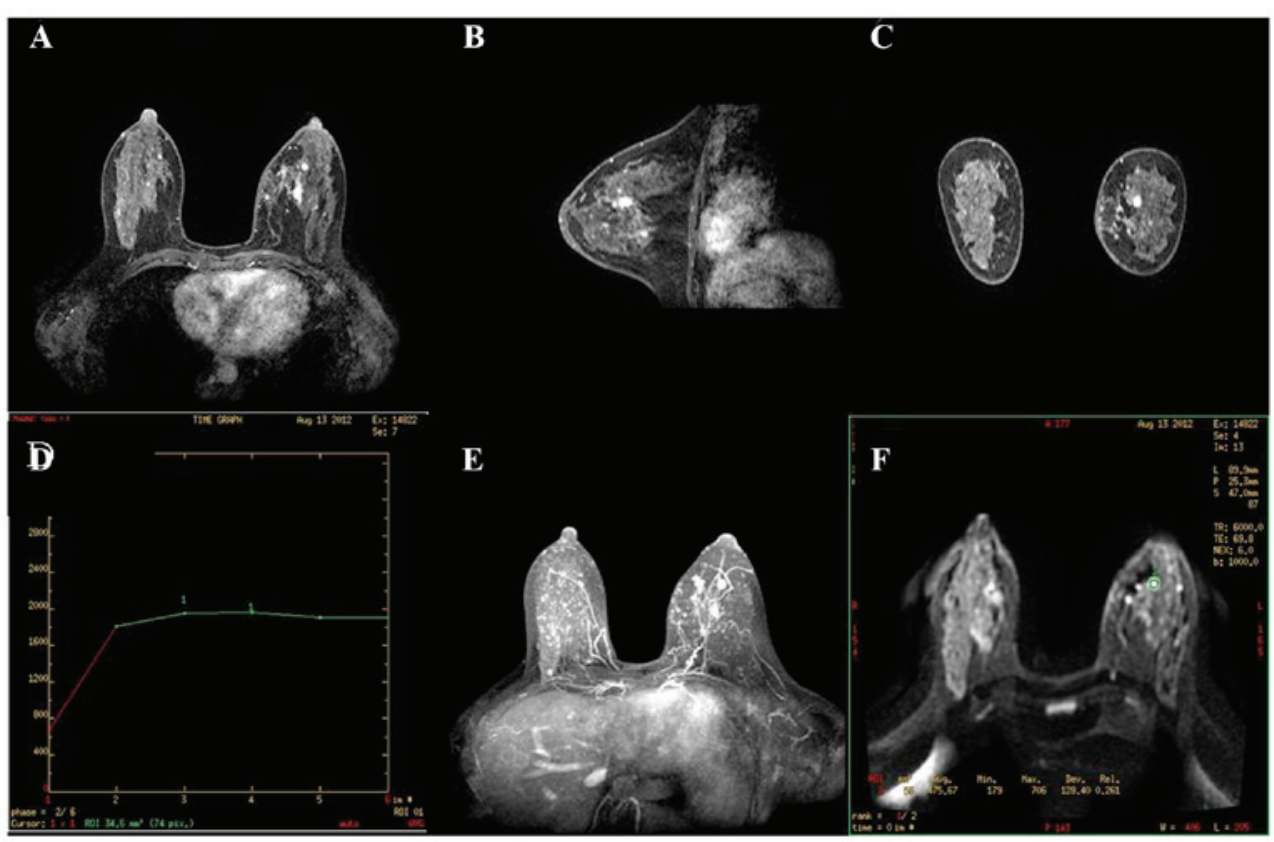

Figure 4. Magnetic resonance images of case no. 4, a 30-year-old with non-specific invasive ductal carcinoma grade II. (A, B and C) Vibrant+C T1 sequence with fat suppression showed multinodular enhancement. (D) Curve of enhancement was plateau-shaped (type II). (E) 3D maximum intensity projection reconstruction image showed multinodular hyperintense enhancement, as well as increased disorders of the vasculature, which had entered the lesions in the left breast. (F) Echo-planar imaging-DWI showed a hypointense lesion in DWI $(b=1,000)$ and the apparent diffusion coefficient was $0.88-1.07 \times 10^{-3} \mathrm{~mm}^{2} / \mathrm{sec}$. DWI, diffusion-weighted imaging.

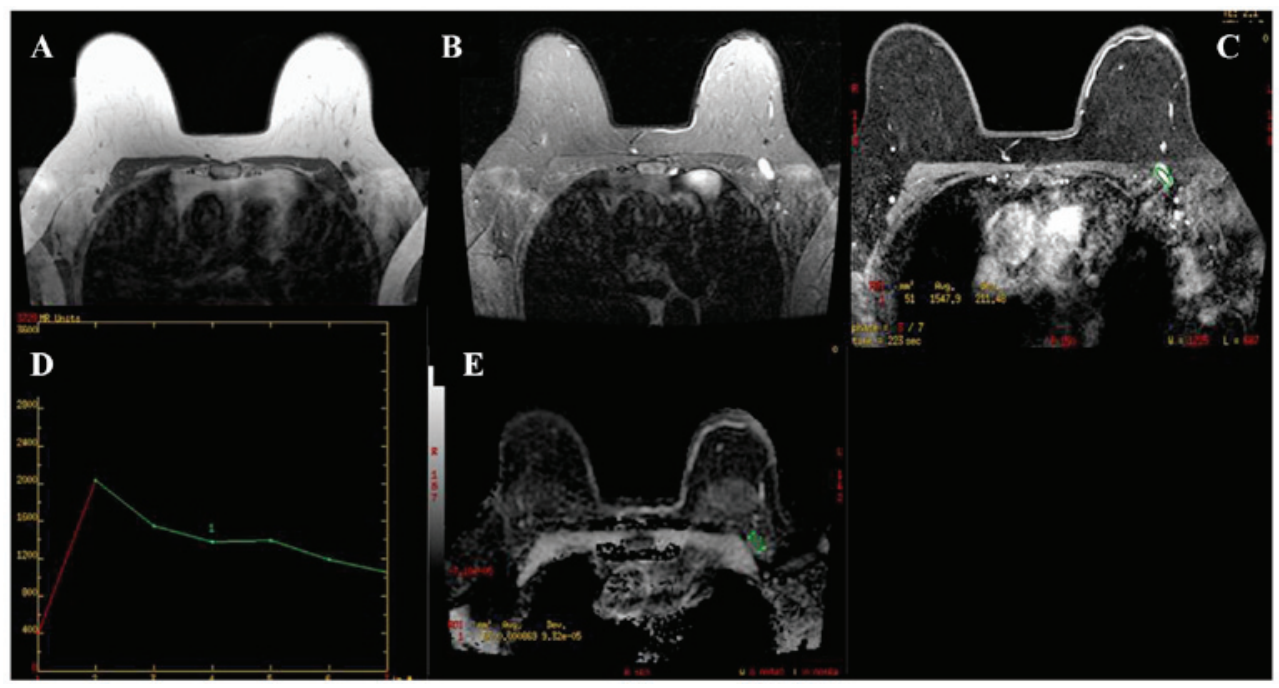

Figure 5. Magnetic resonance images of case no. 4, a 30-year-old with non-specific invasive ductal carcinoma grade II. (A and B) T1-weighted and T2 fat suppression showed an oval-shaped nodule in the left armpit, a homogeneous T1 hypointense signal and a homogeneous T2 fat suppression hyperintense signal. (C) Vibrant+C T1 with fat suppression showed homogeneity enhancement. (D) The curve of enhancement had a washout enhancement pattern (type III). (E) The apparent diffusion coefficient was $0.7 \times 10^{-3} \mathrm{~mm}^{2} / \mathrm{sec}$.

an appropriate surgical approach based on the result of the frozen section diagnosis. Therefore, frozen section pathology is crucial for the diagnosis of breast abnormalities (14).

In the present study, the pathological characteristics of the breast lesions in the majority of patients were typical and a pathological diagnosis was easily made. For certain patients, however, it was not the case. The average rate of correct diagnosis based on frozen section pathology is $92.6-98.0 \%$, and accordingly, the rate of misdiagnosis is $2.0-7.4 \%$. The main reasons of misdiagnosis are as follows: i) Incorrect sampling; ii) poor quality of frozen sections (15); iii) a limited time for pathologist to diagnose; iv) a lack of specific stains and immunohistochemistry; and v) limited experience of the pathologist (16). Misdiagnosis based on intraoperative frozen sections of breast tumors is unavoidable and leads to the selection of an inappropriate surgical plan and causes a psychological, physical and economic burden for patients (17). Therefore, it is important to establish an appropriate therapy plan for patients as soon as possible following identification of the misdiagnosis (18). 
At present, false-negative cases are required to undergo reexcision or even sentinel lymph node biopsy with the smallest possible delay in order to ensure complete resection of the tumor and prevent metastasis to the lymph nodes. Prior to selection of the second surgical scheme, a comprehensive and objective assessment of the postoperative breast is required. However, compared with patients not receiving surgery, breast imaging is more complex for patients who recently underwent surgery. Under such conditions, MRI may be the optimal choice among the three most common types of breast examinations (mammography, ultrasound and MRI) to assess the condition of the postoperative breast (19). MRI has certain advantages: The breast does not require to be compressed in the process of scanning. Furthermore, the scan sequence of MRI is diversified and each sequence may be used for the analysis of lesions from different inspections. Additionally, MRI can help physicians evaluate the presence of multifocal lesions (20). Finally, MRI can predict axillary lymph node metastasis (21). Therefore, the present study retrospectively reviewed the postoperative MRI scans of these patients.

To date, only a small number of studies have reported the use of MRI in the short-term follow-up for the detection of postoperative residual breast cancer. Chae et al (22) reported that dynamic contrast-enhanced breast MRI was a useful tool for residual disease prediction following excisional biopsy for breast cancer. However, the study subjects were patients who underwent excisional biopsy, rather than patients misdiagnosed by intraoperative frozen section pathology. Furthermore, in the present study, MRI findings were analyzed on the basis of contrast-enhanced and DWI scans, while in the study by Chae et al (22), MRI findings were analyzed only on the basis of contrast-enhanced scans.

Among the 10 subjects of the present study, 4 cases with tumor residues and 1 case with axillary lymph node metastasis were diagnosed by MRI and the second pathology. None of the patients received MRI prior to the surgery due to various reasons. However, 1 patient with a tumor residue was misdiagnosed by MRI, following which a correct diagnosis was made based on the pathological findings.

The images of the 4 patients diagnosed as having tumor residues by MRI and pathology were analyzed. Three cases showed nodular enhancement and 1 showed structural disorders with branch-like enhancement. One case showed type-I time-signal intensity curves (TIC) and 3 showed type-II TIC. The ADC value for all 4 cases was $<1 \times 10^{-3} \mathrm{~mm}^{2} / \mathrm{sec}$. These results demonstrated that for breast cancer patients, MRI within 1 month after breast surgery had a high diagnostic capacity in spite of local structural disorders due to surgery.

In the patient misdiagnosed by MRI, no abnormal enhancement was found in the MRI and the ADC value was $1.4-2.0 \times 10^{-3} \mathrm{~mm}^{2} / \mathrm{sec}$. In this case, the information provided by the MRI was inconsistent with the pathological findings, which may be that the pathological type of this patient was neuroendocrine carcinoma. The current consensus is that the majority of cases of neuroendocrine carcinoma are well differentiated and lack typical and evident malignant signs observed by imaging, which may conduce to the misdiagnosis based on MRI (23).

In the present study, based on the results of the postoperative MRI, surgeons adopted two different surgical modes for the
10 patients; 4 were subjected to breast-conserving surgery and 6 accepted modified radical mastectomy. Histological examination of paraffin-embedded tissues following the second surgery showed no residual cancer in any of the 10 patients, who have not presented with any signs of tumor recurrence to date.

In conclusion, the present study suggested that short-term follow-up MRI is of value for detecting postoperative residual breast tumors and may guide surgeons in the selection of the optimal surgical scheme. However, due to the limited number of cases, further studies on a larger patient cohort are required to confirm this conclusion.

\section{References}

1. Murray PJ, Wivell G and Denton E: Breast cancer screening and diagnosis in the 21st century within the UK. Post Reprod Health 21: 105-111, 2015.

2. Pak F, Kanan HR and Alikhassi A: Breast cancer detection and classification in digital mammography based on non-subsampled contourlet transform (NSCT) and Super resolution. Comput Methods Programs Biomed 122: 89-107, 2015.

3. Mann RM,Balleyguier C, Baltzer PA,Bick U, Colin C, Cornford E, Evans A, Fallenberg E, Forrai G, Fuchsjäger MH, et al: Breast MRI: EUSOBI recommendations for women's information. Eur Radiol 25: 3669-3678, 2015.

4. Chetlen A, Mack J and Chan T: Breast cancer screening controversies: Who, when, why, and how? Clin Imaging, 2015 (Epub ahead of print).

5. Giess CS, Yeh ED, Raza S and Birdwell RL: Background parenchymal enhancement at breast MR imaging: Normal patterns, diagnostic challenges and potential for false-positive and false-negative interpretation. Radiographics 34: 234-247, 2014.

6. El-Bolkainy TM, Shabaan HA, Abodeif WT, El-Bolkainy MN and El-Tony A: Intra-operative diagnosis of breast mass-lesions: Comparison of the validity of touch smear preparation and frozen section techniques. J Egypt Natl Canc Inst 20: 63-69, 2008.

7. Niu Y, Fu XL, Yu Y, Wang PP and Cao XC: Intra-operative frozen section diagnosis of breast lesions: A retrospective analysis of 13,243 Chinese patients. Chin Med J (Engl) 120: 630-615, 2007.

8. Wen MC, Chen JT and Ho WL: Frozen-section diagnosis in surgical pathology: A quality assurance study. Kaohsiung J Med Sci 13: 534-539, 1997.

9. Law Y, Cheung PS, Lau S and Lo GG: Impact of magnetic resonance imaging on preoperative planning for breast cancer surgery. Hong Kong Med J 19: 294-299, 2013.

10. Houssami N, Turner R and Morrow M: Preoperative magnetic resonance imaging in breast cancer: Meta-analysis of surgical outcomes. Ann Surg 257: 249-255, 2013.

11. Vilar VS, Goldman SM, Ricci MD, et al: Analysis by MRI of residual tumor after radiofrequency ablation for early stage breast cancer. AJR Am J Roentgenol 198: W285-W291, 2012.

12. Yili Z, Xiaoyan H, Hongwen D, Yun Z, Xin C, Peng W and Youmin G: The value of diffusion-weighted imaging in assessing the ADC changes of tissues adjacent to breast carcinoma. BMC Cancer 9: 18, 2009.

13. Brunner AH, Sagmeister T, Kremer J, Riss P and Brustmann H: The accuracy of frozen section analysis in ultrasound- guided core needle biopsy of breast lesions. BMC Cancer 9: 341, 2009.

14. Bellolio JE, Guzmán GP, Orellana CJ, Roa S JC, Villaseca HM, Araya O JC, Tapia EO and Ineda NV: Diagnostic value of frozen section biopsy during surgery for breast lesions or neoplasms. Rev Med Chil 137: 1173-1178, 2009 (In Spanish).

15. Geramizadeh B, Larijani TR, Owji SM, Attaran SY, Torabinejad S, Aslani FS, Monabati A, Kumar PV and Tabei SZ: Accuracy of intra-operative frozen section consultation in south of Iran during four years. Indian J Pathol Microbiol 53: 414-417, 2010.

16. Brun JL, Cortez A, Rouzier R, Callard P, Bazot M, Uzan S and Daraï E: Factors influencing the use and accuracy of frozen section diagnosis of epithelial ovarian tumors. Am J Obstet Gynecol 199: 244.e1-e7, 2008.

17. Kim JH, Kim TJ, Park YG, Lee SH, Lee CW, Song MJ, Lee KH, Hur SY, Bae SN and Park JS: Clinical analysis of intra-operative frozen section proven borderline tumors of the ovary. Gynecol Oncol 20: 176-180, 2009. 
18. Slama J, Dundr P, Dusek L and Cibula D: High false negative rate of frozen section examination of sentinel lymph nodes in patients with cervical cancer. Gynecol Oncol 129: 384-388, 2013.

19. Saunders C and Taylor D: Expanding the indications for MRI in the diagnosis and treatment of breast cancer: What is best practice? J Med Radiat Sci 62: 47-53, 2015

20. Almotairi M, Oudjhane K and Chavhan GB: Pediatric multifocal liver lesions evaluated by MRI. Indian J Radiol Imaging 25 296-302, 2015.

21. Kuijs VJ, Moossdorff M, Schipper RJ, Beets-Tan RG, Heuts EM Keymeulen KB, Smidt ML and Lobbes MB: The role of MRI in axillary lymph node imaging in breast cancer patients: A systematic review. Insights Imaging 6: 203-215, 2015.
22. Chae EY, Cha JH, Kim HH, et al: Evaluation of residual disease using breast MRI after excisional biopsy for breast cancer. AJR Am J Roentgenol 200: 1167-1173, 2013

23. Rovera F, Masciocchi P, Coglitore A, La Rosa S, Dionigi G, Marelli M, Boni L and Dionigi R: Neuroendocrine carcinomas of the breast. Int J Surg 6 (Suppl 1): S113-S115, 2008. 